ECCOMAS Proceedia
COMPDYN 2017

$6^{\text {th }}$ ECCOMAS Thematic Conference on Computational Methods in Structural Dynamics and Earthquake Engineering M. Papadrakakis, M. Fragiadakis (eds.) Rhodes Island, Greece, 15-17 June, 2017

\title{
LOW-FREQUENCY VIBRATIONS OF THIN CYLINDRICAL SHELL STIFFENED BY RINGS WITH T-SHAPED CROSS-SECTION
}

\author{
Sergei B. Filippov \\ St. Petersburg State University \\ 7/9 Universitetskaya emb., St. Petersburg, 199034, Russia \\ e-mail:s_b_filippov@mail.ru
}

Keywords: Free vibrations, Ring-stiffened shell, T-shaped ring cross-section, Eigenvalue problem, Asymptotic methods.

\begin{abstract}
Small free low-frequency vibrations of a thin elastic cylindrical shell stiffened by the rings with $T$-shaped cross-section are analyzed. The narrow rings are considered as circular beams. The wide rings are treated as annular plates stiffened at one edge by a circular beam. In the both cases problem is reduced to solution of the eigenvalue problem for systems of linear differential equations containing a small parameter. By means of various asymptotic approaches simple approximate formulas for the low frequencies are obtained. These formulas are used for the evaluation of optimal parameters corresponding to the maximal fundamental frequency of the ring-stiffened shell with a given mass.
\end{abstract}




\section{INTRODUCTION}

Ring-stiffened cylindrical shells are widely applied in engineering. Often the rings with rectangular or T-shape cross-sections are used ([1], [2]). The vibrations of the shell stiffened by the rings with rectangular cross-sections are sufficiently well studied e.g. in [3], [4], [5] and [6]. In this paper the shell stiffened by rings with T-shape cross-sections is considered. In many cases the use of the rings with T-shape cross-sections instead of the rings with rectangular crosssections allows to increase the low frequencies of a ring-stiffened shell without the increase in its weight.

Differential equations describing vibrations of a thin shells contain the dimensionless shell thickness as a small parameter. By means of asymptotic approaches ([7], [8], [9]) complex eigenvalue problems of the stiffened shells theory can be transformed into problems which have simple analytical solutions.

If the width of the rings is sufficiently small the typical vibration mode is similar to the vibration mode of the shell without rings, and the shell surface is covered by a series of pits stretched along the generatrix of the cylinder. In this case, considering the rings as circular beams, it is possible to seek solutions as a sum of slowly varying functions and boundary layers near shell edges and stiffened parallels. In the first approximation one get eigenvalue problem describing also vibrations of a beam stiffened by springs. If the rings are identical and uniformly arranged along the shell then a solution of the last problem can be obtained in a closed form by means of a homogenization procedure ([6]).

If the width of a ring grows, the low frequencies increase until the typical vibration mode will be replaced with the axisymmetric vibration mode localized on the surface of the ring while the cylindrical shell itself does not actually deform. At the further increase in width of a ring the low frequencies decrease ([10]). The beam model can not be used for studying vibrations of the wide ring. The ring with T-shape cross-section may be considered as an annular plate stiffened by a circular beam with the rectangular cross-section. An asymptotic analyze shows that the couple vibration problem for the cylindrical shell joined with the annular plate in the first approximation reduce to the eigenvalue problem describing vibrations of the plate. In assumption that the ratio of the plate width to the radius of its inner edge is small, a simple approximate equation for the low frequencies is obtained.

\section{BASIC EQUATIONS}

Consider the low-frequency free vibrations of a thin circular cylindrical shell stiffened by $n_{r}$ identical rings at the parallels $s=s_{i}$, where $s \in[0, l]$ is the dimensionless coordinate in the longitudinal directions, $i=1,2, \ldots n_{r}$ (see Fig. 1). The radius $R$ of the cylindrical shell is

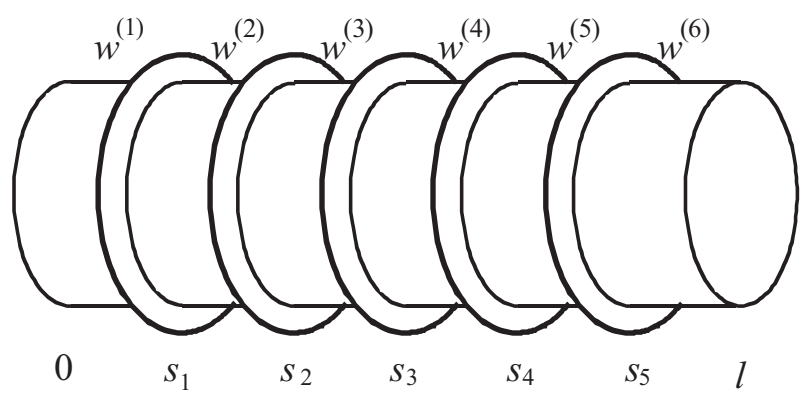

Figure 1: Ring-stiffened cylindrical shell. 
taken as the characteristic size.

After the separation of variables the non-dimensional equations describing free vibrations of a cylindrical shell can be written in the following form

$$
\begin{gathered}
T_{1}^{\prime}+m S+\lambda u=0, \quad S^{\prime}-m T_{2}+Q_{2}+\lambda v=0, \\
Q_{1}^{\prime}+m Q_{2}-T_{2}+\lambda w=0, \quad Q_{1}=M_{1}^{\prime}+2 m H, \quad Q_{2}=-m M_{2}, \\
T_{1}=\varepsilon_{1}+\nu \varepsilon_{2}, \quad T_{2}=\varepsilon_{2}+\nu \varepsilon_{1}, \quad S=(1-\nu)\left(v^{\prime}-m u\right) / 2, \\
M_{1}=\mu^{4}\left(\vartheta_{1}^{\prime}+\nu m \vartheta_{2}\right), \quad M_{2}=\mu^{4}\left(m \vartheta_{2}+\nu \vartheta_{1}^{\prime}\right), \quad H=\mu^{4}(1-\nu) \vartheta_{2}^{\prime}, \\
\varepsilon_{1}=u^{\prime}, \quad \varepsilon_{2}=m v+w, \quad \vartheta_{1}=-w^{\prime}, \quad \vartheta_{2}=m w+v .
\end{gathered}
$$

where $\left({ }^{\prime}\right)$ denotes the derivative with respect to the longitudinal coordinate $s, m$ is the circumferential wave number, $u, v, w$ are the components of the displacement, $\lambda=\sigma \rho \omega^{2} R^{2} E^{-1}$ is the frequency parameter, $\sigma=1-\nu^{2}, \nu$ is Poisson's ratio, $E$ is Young's modulus, $\rho$ is the mass density, $\omega$ is the vibration frequency, $T_{1}, T_{2}, S, Q_{1}, Q_{2}, M_{1}, M_{2}, H$ are the dimensionless stress-resultants and stress-couples, $\vartheta_{1}$ and $\vartheta_{2}$ are the angles of rotation of the normal, $\mu$ is the small parameter, $\mu^{4}=h^{2} / 12, h$ is the dimensionless shell thickness.

We denote as $y^{(j)}$ the solutions of equations (1) in the intervals $s \in\left[s_{j-1}, s_{j}\right], j=1,2, \ldots n$, $n=n_{r}+1$. These solutions satisfy 8 boundary conditions on the shell edges $s=0$ and $s=l$ and $8 n_{r}$ continuity conditions on the parallels $s=s_{i}, i=1,2, \ldots, n_{r}$.

\section{FIRST APPROXIMATION FOR NARROW RINGS}

Assume that the width of the rings is sufficiently small. Then the lowest eigenvalues $\lambda \sim$ $\mu^{2}$ corresponds to the large circumferential wave number $m \sim \mu^{-1 / 2}$ and we seek unknown functions in equations (1) as

$$
y^{(j)}(s)=y_{0}^{(j)}(s)+\sum_{k=1}^{4} D_{k}^{(j)} Y_{k}^{(j)}(s), \quad s \in\left[s_{i-j}, s_{j}\right], \quad j=1,2, \ldots, n .
$$

Here $y_{0}^{(j)}$ are slowly varying functions, $D_{k}^{(j)}$ are arbitrary constants. The edge effect functions $Y_{k}^{(j)}$, are localized in a small neighborhoods of the parallels $s_{0}, s_{1}, \ldots, s_{n}$.

The equations of the first approximation are

$$
\frac{d^{4} w_{0}^{(j)}}{d s^{4}}-\alpha^{4} w_{0}^{(j)}=0, \quad \sigma \alpha^{4}=m^{4} \lambda-\mu^{4} m^{8}, \quad j=1,2, \ldots, n .
$$

System of equations (3) has the order $4 n$ and its solutions can not satisfy all $8 n$ boundary conditions of the initial eigenvalue problem. The problem of the extracting the boundary conditions for equations (3) out of the boundary conditions for equations (1) is discussed in [9].

The solutions of equations (3) must satisfy $4 n_{r}$ continuity conditions on the parallels $s=s_{i}$ :

$$
\begin{aligned}
& w_{0}^{(i)}=w_{0}^{(i+1)}, \quad w_{0}^{(i) \prime}=w_{0}^{(i+1) \prime}, \quad w_{0}^{(i) \prime \prime}=w_{0}^{(i+1) \prime \prime}, \quad w_{0}^{(i) \prime \prime \prime}-w_{0}^{(i+1) \prime \prime \prime}=-c w_{0}^{(i+1)}, \\
& c=m^{8} I / h \text {, }
\end{aligned}
$$

where $I$ is the dimensionless moment of inertia of a ring cross-section. The boundary conditions for equations (3) at the freely supported shell edges have the form

$$
w_{0}^{(1)}(0)=w_{0}^{(1) \prime \prime}(0)=w_{0}^{(n)}(l)=w_{0}^{(n) \prime \prime}(l)=0 .
$$


The eigenvalue problem (3-5) also describe the flexural vibrations of the simply supported beam, stiffened by $n_{r}$ identical springs of the stiffness $c$ at the points $s=s_{i}$.

From the second formula (3) it follows that the approximate values of the frequency parameter $\lambda$ are

$$
\lambda_{k}(m)=\sigma \alpha_{k}^{4}(m) / m^{4}+\mu^{4} m^{4}, \quad k=1,2, \ldots,
$$

where $0<\alpha_{1} \leq \alpha_{2} \leq \cdots$ are eigenvalues of the eigenvalue problem (3-5). The minimal frequency parameter $\lambda_{1}$, corresponding to the fundamental frequency $\omega_{1}$, can be obtained by the formula

$$
\lambda_{1}=\min _{m}\left[\sigma \alpha_{1}^{4}(m) / m^{4}+\mu^{4} m^{4}\right] .
$$

Assume that rings are uniformly arranged, i. e. $s_{i}=i l / n$, the number of rings $n_{r}$ is large and the stiffness $c$ of rings is small. In this case one can use the homogenization method [6] for the approximate evaluation of the eigenvalues $\alpha_{k}$. Instead of the eigenvalue problem (3-5) we will solve the equivalent problem for the equation

$$
\frac{d^{4} w}{d s^{4}}+c w \sum_{i=1}^{n_{r}} \delta\left(s-s_{i}\right)=\alpha^{4} w
$$

with the boundary conditions (5). Here $\delta(z)$ is Dirac's delta function. In the new variables $s=x l$ and $w=\hat{w} l$ equation (7) is

$$
\frac{d^{4} \hat{w}}{d x^{4}}+\hat{c} n \hat{w} \sum_{i=1}^{n_{r}} \delta(\xi-i)=\kappa \hat{w}
$$

where $\hat{c}=c l^{3}, \kappa=(\alpha l)^{4}$, and $\xi=n x$. Boundary conditions (5) take the form

$$
\hat{w}=\frac{d^{2} \hat{w}}{d x^{2}}=0, \quad x=0, \quad x=1 .
$$

Assuming that $n \gg 1$ and $\hat{c} n \sim 1$, we seek the solution of equation (8) as

$$
\hat{w}(x, \xi)=w_{0}(x, \xi)+n^{-4} w_{4}(x, \xi)+\cdots, \quad \kappa=\kappa_{0}+n^{-4} \kappa_{4}+\cdots,
$$

where $w_{i}(x, \xi)=w_{i}(x, \xi+1)$ and, since,

$$
<\frac{\partial^{k} w_{i}}{\partial \xi^{k}}>=\int_{\xi}^{\xi+1} \frac{\partial^{k} w_{i}}{\partial \xi^{k}} d \xi=0, \quad i=0,4, \ldots, \quad k=1,2, \ldots
$$

The operator $\langle\cdot\rangle$ is called the homogenization operator. The application of this operator to both parts of an equation is called the homogenization of the equation.

Substituting (10) into (8), we obtain the equations

$$
\frac{\partial^{4} w_{0}}{\partial \xi^{4}}=0, \quad \frac{\partial^{4} w_{4}}{\partial \xi^{4}}+\frac{\partial^{4} w_{0}}{\partial x^{4}}+\hat{c} n \sum_{i=1}^{n} \delta(\xi-i) w_{0}=\kappa_{0} w_{0} .
$$

From the first of equations (11) it follows that

$$
\frac{\partial^{3} w_{0}}{\partial \xi^{3}}=v_{3}(x), \quad v_{3}(x)=<v_{3}(x)>=<\frac{\partial^{3} w_{0}}{\partial \xi^{3}}>=0 .
$$


A further integration followed by a homogenization gives

$$
\frac{\partial^{2} w_{0}}{\partial \xi^{2}}=v_{2}(x)=0, \quad \frac{\partial w_{0}}{\partial \xi}=v_{1}(x)=0, \quad w_{0}(x, \xi)=v_{0}(x) .
$$

After the homogenization of the second of equations (11) we get

$$
\frac{d^{4} v_{0}}{d x^{4}}+\hat{c} n v_{0}=\kappa_{0} v_{0}
$$

The boundary conditions for equation (12) are

$$
v_{0}=\frac{d^{2} v_{0}}{d x^{2}}=0, \quad x=0, \quad x=1 .
$$

Eigenvalue problem (12), (13) describes the vibrations of a simply supported beam on an elastic base and has the solutions $v_{0 k}=\sin (k \pi x)$,

$$
\kappa_{0 k}=(k \pi)^{4}+\hat{c} n, \quad k=1,2, \ldots
$$

Although formula (14) is derived for $n \gg 1$ and $\hat{c} \sim 1 / n \ll 1$, they provide good approximations for the exact values of $\kappa_{1}$ even for $n=2$ (for one ring) and for a sufficiently large stiffness $\hat{c}$ (see [6]). It follows from (14) that

$$
\alpha_{k}^{4}=\frac{\kappa_{k}}{l^{4}}=\frac{\pi^{4} k^{4}}{l^{4}}+\frac{c n}{l}=\frac{\pi^{4} k^{4}}{l^{4}}+\frac{\eta \mu^{4} m^{8}}{\sigma}, \quad \eta=\frac{\sigma c n}{m^{8} \mu^{4} l}=\frac{\sigma n I}{\mu^{4} h l} .
$$

The relative ring stiffness $\eta$ is proportional to the ratio $D_{r} / D$, where $D_{r}=E I$ is the bending stiffness of the ring and $D=E h^{3} /(12 \sigma)$ is the bending stiffness of the shell.

Substituting (15) into (6) we get the following approximate formula

$$
\lambda_{1}^{*}(\eta)=\lambda_{1}^{*}(0) \sqrt{1+\eta}
$$

where $\lambda_{1}^{*}(\eta)$ and $\lambda_{1}^{*}(0)=2 \sqrt{\sigma} \mu^{2} \pi^{2} / l^{2}$ are the approximate values of frequency parameters for the ring-stiffened and non-stiffened cylindrical shells accordingly.

The lowest frequency parameter $\lambda_{1}$, corresponding to fundamental frequency, is the important characteristics of a shell. For a non-stiffened cylindrical shell the approximate value of $\lambda_{1}$ can be found by the formula $\lambda_{1} \simeq \lambda_{1}^{*}(0)$. However, for the ring-stiffened cylindrical shell relation $\lambda_{1} \simeq \lambda_{1}^{*}(\eta)$ is valid only if the relative ring stiffness $\eta$ is not to large, because eigenvalue problem (3-5) in case $s_{j}=j l / n$ has stiffness-independent solutions $w_{0}=\sin (n \pi s / l)$, $\alpha_{0}=\pi n / l$. Corresponding to this solution the minimal stiffness-independent eigenvalue is $\lambda_{n}^{*}(0)=n^{2} \lambda_{1}^{*}(0)$.

The root of the equation $\lambda_{1}^{*}(\eta)=\lambda_{n}^{*}(0)$ is $\eta^{*}=n^{4}-1$. If $\eta \leq \eta^{*}$ then $\lambda_{1}^{*}(\eta) \leq \lambda_{n}^{*}(0)$ else $\lambda_{1}^{*}(\eta)>\lambda_{n}^{*}(0)$. Therefore, for ring-stiffened shell

$$
\lambda_{1}(\eta) \simeq \begin{cases}\lambda_{1}^{*}(0) \sqrt{1+\eta)}, & 0 \leq \eta \leq \eta^{*} \\ n^{2} \lambda_{1}^{*}(0), & \eta>\eta^{*}\end{cases}
$$




\section{OPTIMAL DESIGN}

We suppose that the mass $M_{s}$ of a ring-stiffened simply supported cylindrical shell is given and seek the optimal parameters for which its fundamental vibrations frequency $\omega_{1}$ has the largest value.

The fundamental vibration frequency $\omega_{0}$ of the unstiffened simply supported cylindrical shell of the thickness $h_{0}$ and length $l$ can be found by the approximate formula

$$
\omega_{0}^{2}=\frac{E \lambda_{1}(0)}{\sigma \rho R^{2}}=\frac{E h_{0} \pi^{2}}{\sqrt{3 \sigma} \rho R^{2} l^{2}} .
$$

The mass of the unstiffened shell $M_{0}=2 \pi \rho l h_{0} R^{3}$. Assume that $M_{s}=M_{0}$. The values of the ring-stiffened shell parameters for which the ratio $f=\omega_{1} / \omega_{0}$ attains its maximum are called its optimal values.

The approximate values of optimal parameters for the cylindrical shell stiffened by the rings with rectangular cross-sections are obtained in [6]. Consider the cylindrical shell stiffened by the rings with T-shaped cross-sections. The dimensionless sizes of the T-shaped cross-sections are shown in Fig. 2.

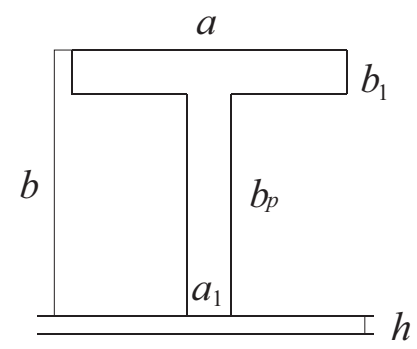

Figure 2: T-shaped cross-sections of the ring.

The mass of a cylindrical shell stiffened by the rings with the T-shaped cross-sections is $M_{s}=2 \pi R^{3} \rho\left(h l+n_{r} S\right)$, where $S=a^{2} k\left(k_{a}+k_{b}-k_{a} k_{b}\right)$ is the area of the ring cross-section, $k=b / a, k_{a}=a_{1} / a, k_{b}=b_{1} / b$. If $k_{a}=1$ or $k_{b}=1$ then the cross-section is the rectangle and $S=a^{2} k$. It follows from the condition $M_{s}=M_{0}$ that

$$
z=1-A a^{2}, \quad A=\frac{n_{r} k\left(k_{a}+k_{b}-k_{a} k_{b}\right)}{l h_{0}},
$$

where $z=h / h_{0}, h$ is the thickness of the ring-stiffened shell.

Using formula (17) we obtain

$$
f^{2}=\frac{\omega_{1}^{2}}{\omega_{0}^{2}}= \begin{cases}z \sqrt{1+\eta)}, & 0 \leq \eta \leq \eta^{*}, \\ z n^{2}, & \eta>\eta^{*},\end{cases}
$$

The moment of inertia of T-shaped cross-section with respect to the generatrix of the cylinder is

$$
I=a^{4} k^{3}\left[1+\left(1-k_{a}\right)\left(1-k_{b}\right)^{3}\right] / 3 .
$$

Taking into account that

$$
\eta=\frac{12 \sigma n I}{h^{3} l}=\frac{B a^{4}}{z^{3}}=\frac{B(1-z)^{2}}{A^{2} z^{3}}, \quad B=\frac{4 \sigma n k^{3}\left[1+\left(1-k_{a}\right)\left(1-k_{b}\right)^{3}\right]}{h_{0}^{3} l},
$$


from relation (20) we obtain

$$
f^{2}(z)= \begin{cases}z n^{2}, & z \leq z^{*} \\ z \sqrt{1+\gamma(z-1)^{2} / z^{3}}, & z>z^{*}\end{cases}
$$

where $\gamma=B / A^{2}$ and $z^{*} \in(0,1)$ is the root of the cubic equation

$$
g(z)=z^{3}-q(z-1)^{2}=0, \quad q=\gamma / \eta^{*} .
$$

By virtue of the inequalities $g(1)=1>0$ and $g(0)=-q<0$ the function $g(z)$ has at least one root in the interval $(0,1)$. This root is unique, since

$$
g^{\prime}=d g / d z=3 z^{2}-2 q(z-1)>0 \quad \text { for } \quad z \in[0,1] .
$$

As was to be proved in [6] for the small values of $h_{0}$

$$
f^{*}=\max _{0<z<1} f(z)=f\left(z^{*}\right)=n \sqrt{z^{*}},
$$

and the optimal values of the parameters $z$ and $a$ are $z^{*}$ and $a^{*}=\sqrt{\left(1-z^{*}\right) / A}$.

Let $l=4, h_{0}=0.01, \nu=0.3$. The values of $z^{*}, a^{*}$ and $f^{*}$ for various numbers of rings $n_{r}$ are given in Table 1. In left and right parts of the table are listed the results for the rings with square cross-section $\left(k=1, k_{a}=1\right)$ und T-shaped cross-section $\left(k=1, k_{a}=k_{b}=0.2\right)$ correspondingly. From Table 1 it follows that the replacement of the rings with the square cross-

\begin{tabular}{c|ccc|ccc}
\hline \multirow{2}{*}{$n_{r}$} & \multicolumn{3}{|c|}{$k=1, k_{a}=k_{b}=1$} & \multicolumn{3}{c}{$k=1, k_{a}=k_{b}=0.2$} \\
\cline { 2 - 7 } & $z^{*}$ & $a^{*}$ & $f^{*}$ & $z^{*}$ & $a^{*}$ & $f^{*}$ \\
\hline 1 & 0.935 & 0.0510 & 1.934 & 0.968 & 0.0596 & 1.968 \\
2 & 0.805 & 0.0625 & 2.691 & 0.893 & 0.0771 & 2.835 \\
4 & 0.537 & 0.0680 & 3.667 & 0.687 & 0.0932 & 4.145 \\
6 & 0.363 & 0.0652 & 4.218 & 0.507 & 0.0955 & 4.985 \\
10 & 0.192 & 0.0586 & 4.826 & 0.292 & 0.0887 & 5.947 \\
\hline
\end{tabular}

Table 1: Values of optimal parameters $z^{*}, a^{*}$ and ratio $f^{*}$ vs. number of rings $n_{r}$.

sections to the rings with the T-shaped cross-sections leads to an increase in the fundamental frequency of the stiffened shell without the increase in its mass. For ten rings this increase reaches $23 \%$.

Using formulas (19), (21) and (22) we obtain

$$
\begin{gathered}
A=A_{1} k, \quad A_{1}>0, \quad B=B_{1} k^{3}, \quad B_{1}>0, \quad q=\frac{B}{\eta^{*} A^{2}}=\frac{B_{1} k}{\eta^{*} A_{1}^{2}}, \quad \frac{d q}{d k}=\frac{B_{1}}{\eta^{*} A_{1}^{2}}>0, \\
\frac{d q}{d z}=\frac{z^{2}(3-z)}{(1-z)^{3}}>0 .
\end{gathered}
$$

Therefore,

$$
\frac{d f^{*}}{d k}=\frac{n}{2 \sqrt{z}} \frac{d z}{d k}=\frac{n}{2 \sqrt{z}} \frac{d z}{d q} \frac{d q}{d k}>0,
$$

and the function $f^{*}(k)$ increases with $k$. However, for large values of $k$ formula (23) is not valid because a wide ring must be considered as an annular plate. 


\section{FIRST APPROXIMATION FOR WIDE RINGS}

We consider the wide rings with T-shaped cross-section as annular plates of the thickness $h_{p}=a_{1}$ and the width $b_{p}=b-b_{1}$ stiffened at one edge by a circular beam. The beam has the rectangular cross-section of the sizes $a$ and $b_{1}$ (see Fig. 2).

The results presented in [10] show that the low-frequency vibrations of the cylindrical shell stiffened by annular plates are axisymmetric, i.e. $m=0$. The equations

$$
\begin{gathered}
T_{1}^{\prime}+\lambda u=0, \quad Q_{1}^{\prime}-T_{2}+\lambda w=0, \quad Q_{1}=M_{1}^{\prime}, \\
T_{1}=u^{\prime}+\nu w, \quad T_{2}=w+\nu u^{\prime}, \quad M_{1}=\mu^{4} \vartheta^{\prime}, \quad \vartheta_{1}=-w^{\prime},
\end{gathered}
$$

describing axisymmetric vibrations of a cylindrical shell are obtained from equations (1).

The non-dimensional equations

$$
\begin{gathered}
\left(s_{p} Q_{1 p}\right)^{\prime}+\lambda s_{p} w_{p}=0, \quad s_{p} Q_{1 p}=\left(s_{p} M_{1 p}\right)^{\prime}-M_{2 p}, \\
s_{p} M_{1 p}=h_{p}^{2}\left(s_{p} \vartheta_{1 p}^{\prime}+\nu \vartheta_{1 p}\right) / 12, \quad s_{p} M_{2 p}=h_{p}^{2}\left(\vartheta_{1 p}+\nu s_{p} \vartheta_{1 p}^{\prime}\right) / 12, \quad \vartheta_{1 p}=-w_{p}^{\prime} \\
\left(s_{p} T_{1 p}\right)^{\prime}-T_{2 p}+\lambda s_{p} u_{p}=0, \quad s_{p} T_{1 p}=s_{p} u_{p}^{\prime}+\nu u_{p}, \quad s_{p} T_{2 p}=u_{p}+\nu s_{p} u_{p}^{\prime},
\end{gathered}
$$

describe the axisymmetric vibrations of the annular plate. Here $\left(^{\prime}\right)$ denotes the derivative with respect to the radial coordinate $s_{p} \in\left[1,1+b_{p}\right], w_{p}$ and $u_{p}$ are the components of the displacement, $Q_{1 p}, T_{1 p}, T_{2 p}$ and $M_{1 p}, M_{2 p}$ are the dimensionless stress-resultant and the stress-couples, $\vartheta_{1 p}$ is the angle of rotation.

For the simply supported shell

$$
u^{\prime}=w=w^{\prime \prime}=0, \quad x=0, \quad x=l .
$$

At the parallel $s=s_{i}$ the following 9 continuity conditions have to be satisfied

$$
\begin{aligned}
& w^{(i)}=w^{(i+1)}=-u_{p}^{(i)}, \quad u^{(i)}=u^{(i+1)}=w_{p}^{(i)}, \quad \vartheta^{(i)}=\vartheta^{(i+1)}=\vartheta_{p}^{(i)}, \\
& h\left(T_{1}^{(i+1)}-T_{1}^{(i)}\right)=h_{p} Q_{1 p}^{(i)}, \quad h\left(M_{1}^{(i+1)}-M_{1}^{(i)}\right)=h_{p} M_{1 p}^{(i)}, \quad h\left(Q_{1}^{(i+1)}-Q_{1}^{(i)}\right)=-h_{p} T_{1 p}^{(i)},
\end{aligned}
$$

where $u_{p}^{(i)}, w_{p}^{(i)}, \vartheta_{p}^{(i)}, \ldots$ are the solutions of equations (25) and (26) for the plate located on parallel with the coordinate $s=s_{i}$. At the outer plate edge $s_{p}=1+b_{p}$, stiffened by circular beam, following 3 conditions should be fulfilled

$$
T_{1 p}^{(i)}=a b_{1} w_{p}^{(i)}, \quad M_{1 p}^{(i)}=c_{b} w_{p}^{(i) \prime}, \quad Q_{1 p}^{(i)}=0, \quad c_{b}=\frac{\sigma a^{3} b_{1}}{h_{p}^{3}},
$$

where $c_{b}$ is the relative stiffness of the beam.

We assume that $h_{p} \sim h, \lambda \sim h_{p}^{2}$ and seek the solutions of equation (24) in the form (2). In the first approximation $w_{0}^{(j)}=u_{0}^{(j)}=u_{p}^{(i)}=0$ and the eigenvalue problem (24-29) reduced to the solution of equation (25) with the boundary conditions

$$
w_{p}=\vartheta_{1 p}=0, \quad s_{p}=1, \quad M_{1 p}=c_{b} w_{p}^{\prime} \quad Q_{1 p}=0, \quad s_{p}=1+b_{p} .
$$

Assume that the plate is narrow, i.e. $b_{p} \ll 1$. Replacing variable $s=1+b_{p} x$ in the equations (25) and neglecting small terms leads to the approximate equation (see [11])

$$
\frac{d^{4} w}{d x^{4}}-\beta^{4} w=0, \quad x \in[0,1]
$$


where $\beta^{4}=12 b_{p}^{4} \lambda / h_{p}^{2}$. The solution of equation (31) has the form

$$
w=C_{1} \sin \beta x+C_{2} \cos \beta x+C_{3} \sinh \beta x+C_{4} \cosh \beta x .
$$

Instead boundary conditions (30) we obtain the approximate boundary conditions

$$
w=w^{\prime}=0, \quad x=0, \quad w^{\prime \prime}+c_{b} w^{\prime}=0, \quad w^{\prime \prime \prime}=0, \quad x=1,
$$

Substituting solution (32) into boundary conditions (33) we get four linear homogeneous algebraic equations for unknowns $C_{1}, C_{2}, C_{3}$ and $C_{4}$. This system of equations has nontrivial solutions if its characteristic determinant $D \beta$ ) is equal to zero, i. e.

$$
D(\beta)=\cosh \beta \cos \beta+1+c_{b}(\cosh \beta \sin \beta+\sinh \beta \cos \beta)=0 .
$$

The minimal frequency parameter $\lambda_{1}$, corresponding to fundamental frequency $\omega_{1}$, can be found by following approximate formula

$$
\lambda_{1}=\frac{h_{p} \beta_{1}^{4}}{12 b_{p}^{4}}=\frac{h_{p} \beta_{1}^{4}}{12\left(b-b_{1}\right)^{4}} .
$$

where $\beta_{1}$ is the minimal positive root of equation (34). The values of the root $\beta_{1}$ vs. the relative stiffness of the beam $c_{b}$ are plotted in Fig. 3. The parameter $\beta_{1}$ and frequency parameter $\lambda_{1}$

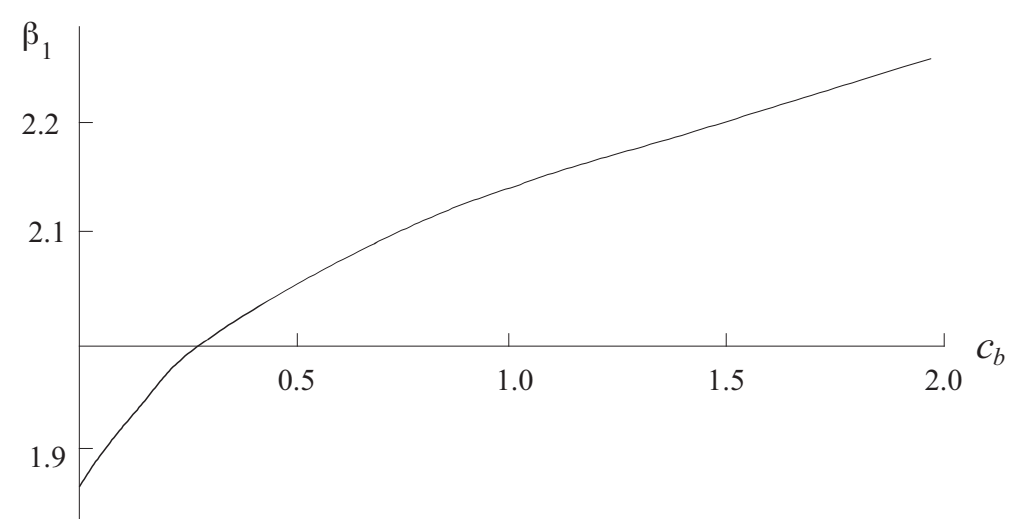

Figure 3: The root $\beta_{1}$ of equation (34) dependent on the stiffness of the beam $c_{b}$.

increase with the beam stiffness $c_{b}$.

According to formula (35), which is valid in the case of wide rings, the minimal frequency parameter $\lambda_{1}$ decreases when the the rings width $b$ increases, while for narrow rings $\lambda_{1}$ increases with $b$.

Let us compare the fundamental frequencies $\omega_{1}^{T}$ and $\omega_{1}^{R}$ of the wide rings with T-shaped and rectangular cross-sections, assuming that the rings have equal width and mass. Suppose that the dimensionless sizes of T-shaped and rectangular cross-sections are $a=0.05, b_{1}=0.01$, $b_{p}^{T}=0.1, h_{p}^{T}=0.1$ and $b_{p}^{R}=b_{p}^{T}+b_{1}=0.11, h_{p}^{R}=\left(h_{p}^{T} b_{p}^{T}+a b_{1}\right) / b^{R}=0.0136$ accordingly. The last approximate formula follows from the equality of the ring masses.

Then $c^{T}=\sigma a^{3} b_{1} /\left(h_{p}^{T}\right)^{3}=1.138, \beta_{1}^{T}=2.16, c^{R}=0, \beta_{1}^{R}=1.87$ and by virtue of the formula (35) we obtain

$$
\frac{\lambda_{1}^{T}}{\lambda_{1}^{R}}=\frac{h_{p}^{T}}{h_{p}^{R}} \frac{\left(\beta_{1}^{T} b_{P}^{R}\right)^{4}}{\left(\beta_{1}^{R} b_{p}^{T}\right)^{4}}=1.91, \quad \frac{\omega_{1}^{T}}{\omega_{1}^{R}}=\sqrt{\frac{\lambda_{1}^{T}}{\lambda_{1}^{R}}}=1.38 .
$$




\section{CONCLUSIONS}

Free low-frequency vibrations of a thin cylindrical shell stiffened by the rings with T-shaped cross-section was analyzed. To obtain simple approximate formulas for the lower frequencies a combination of asymptotic method have been used. The optimal parameters corresponding to the maximal fundamental frequency of the shell with given mass was evaluated. It was shown that the replacement of the rings with the rectangular cross-section to the rings with the T-shaped cross-section leads to increase in the fundamental frequency of the ring-stiffened shell without the increase in its mass.

\section{ACKNOWLEDGEMENTS}

This work was supported by RFBR (grant 16-01-00580) which is gratefully acknowledged.

\section{REFERENCES}

[1] J.G. Teng, J.M. Rotter, Buckling of Thin Metal Shells. CRC Press, 2003.

[2] S.S. Gill, The stress analysis of pressure vessels and pressure components. Oxford: Pergamon Press, 1970.

[3] B. Yang, J. Zhou, Analysis of ring-stiffened cylindrical shells. J. of Applied Mechanics, 62, 1005-1014, 1995.

[4] C.T. Loy, K.Y. Lam, Vibration of Cylindrical Shells with Ring Support. International Journal of Mechanical Sciences, 39, 455-471, 1997.

[5] A. Samantaa, M. Mukhopadhyayb, Free vibration analysis of stiffened shells by the finite element technique. European Journal of Mechanics - A/Solids, 23, 159-179, 2004.

[6] S.B. Filippov, Buckling, vibrations and optimal design of ring-stiffened thin cylindrical shells. Advances in Mechanics of Solids, World Scientific Publishing Co Ltd., 17-48, 2006.

[7] S.M. Bauer, S.B. Filippov, A.L. Smirnov, P.E. Tovstik, R. Vaillancourt, Asymptotic methods in mechanics of solids. International Series of Numerical Mathematics, 167, Springer International Publishing Switzerland, 2015.

[8] I.V. Andrianov, J. Awrejcewicz, L.I. Manevitch, Asymptotical mechanics of thin-walled structures. Berlin, New York: Springer-Verlag, 2004.

[9] P.E. Tovstik, A.L. Smirnov, Asymptotic methods in the buckling theory of elastic Shells. World Scientific, Singapore, 2001.

[10] S.B. Filippov, Optimal design of stiffened cylindrical shells based on an asymptotic approach. J. Technishe Mechanik, 24, 221-230, 2004.

[11] S.B. Filippov, M. Kolyada, Numerical and Asymptotic Modeling of Annular Plate Vibrations. I. Dimov, I. Farago, L. Vulkov (Eds.) 5th Conference on Numerical Analysis and Applications (NAA 2012), June, 2012, Lozenetz, Bulgaria, Springer-Verlag Berlin Heidelberg, 295-302, 2013. 